\title{
Modeling Irradiation Creep of Graphite using Rate Theory
}

\author{
Apu Sarkar ${ }^{\S}$, Jacob Eapen ${ }^{\circledR}$, Anant Raj ${ }^{\S}$, K. L. Murty ${ }^{\S}$ and T. D. Burchell ${ }^{\dagger}$ \\ ${ }^{\S}$ Department of Nuclear Engineering, North Carolina State University, Raleigh, NC 27695, USA. \\ ${ }^{\dagger}$ Fusion Materials \& Nuclear Structures, Oak Ridge National Laboratory, Oak Ridge, TN 37831, USA. \\ jacob.eapen@ncsu.edu
}

\begin{abstract}
We have examined irradiation induced creep of graphite in the framework of transition state rate theory. Experimental data for two grades of nuclear graphite (H-337 and AGOT) have been analyzed to determine the stress exponent $(n)$ and activation energy $(Q)$ for plastic flow under irradiation. We show that the mean activation energy $(Q)$ lies between $0.15-0.32 \mathrm{eV}$ with a mean stress-exponent of $1.0 \pm 0.2$. A stress exponent of unity and the unusually low activation energies strongly indicate a diffusive defect transport mechanism for neutron doses in the range of 3 to $4 \times 10^{22} \mathrm{n} / \mathrm{cm}^{2}$.
\end{abstract}

Keywords: Graphite, Creep, Irradiation, Activation energy

\section{Introduction}

Graphite has been used as a moderator and a reactor core structural material since the beginning of the development of nuclear reactors [1]. It is being extensively used in gas-cooled reactors and is currently considered as a potential moderator/structural-material for the next generation very high temperature reactors (VHTRs). The structural graphite components are expected to maintain the structural integrity for the entire lifetime of the reactor, which typically spans between 40 to 60 years. During reactor operation, the graphite components are subjected to moderate to high temperatures, thermo-mechanical stresses, and fast neutron flux, all of which, have a significant effect on the dimensions and properties of the components. Aided by the high temperatures, the accumulated stresses from irradiation are relieved through irradiation creep that results in dimensional changes of the graphitic components. While stress relaxation is desirable, the dimensional changes due to creep are limited by the operational and safety design constraints. Thus a mechanistic understanding of creep of graphite is critical for predicting the creep strain induced by irradiation at temperatures, stresses and fast neutron fluxes that extend beyond the envelope that is currently available [2].

Due to its technological significance, irradiation creep of graphite has been a topic of extensive research for over four decades [3-14]. Based on experiments on different grades of nuclear graphite, various models have been developed over the years; refinements have been made recently to extend their use to VHTR operational conditions $[2,14]$. In this paper, we analyze two sets of historical irradiation creep data from a rate theory perspective. The main objective of our work is to predict an effective activation energy barrier $(Q)$ and a stress exponent $(n)$, which are not known currently, for steady-state irradiation creep conditions. We apply the rate theory formalism to published creep data of two nuclear graphite grades. Interestingly, the mean activation energy for steady-state creep deformation under irradiation is observed to be rather low (less than $0.32 \mathrm{eV}$ ) with a mean stress exponent of $\sim 1$, suggesting a diffusional defect transport mechanism. We note here that our work does not aim to replace the existing models; rather, the rate theory formalism can serve as a complementary approach that can be advantageously employed to reveal potential pathways for irradiation creep deformation mechanisms in graphite.

\section{Background}

The experimentally determined or apparent irradiation creep strain is conventionally defined as

Page 1/15

(C) 2016. This manuscript version is made available under the Elsevier user license

http://www.elsevier.com/open-access/userlicense/1.0/ 
$\varepsilon=\frac{l^{\sigma}-l^{0}}{l_{0}}$

where $l^{\sigma}$ and $l^{0}$ are the linear dimensions of the crept stressed sample, and the crept unstressed sample, respectively; $l_{0}$ denotes the initial uncrept dimension, which is the same for both stressed and unstressed samples. The early theoretical work of Kelly and Foreman [15] suggests a creep model that includes primary transient and secondary steady-state contributions. In this model, the primary plastic deformation occurs through the flow of basal plane dislocations. Small interstitial clusters, typically comprising of $4 \pm 2$ atoms, which are formed between the basal planes act as pinning sites that impede the motion of basal dislocations. Under irradiation, the pinning sites start to grow initially and then saturate to a steady-state value; concomitantly, the initial (primary) creep strain rate falls with increasing pinning sites, and levels off at larger neutron doses. On continued irradiation, the interstitial clusters get destroyed or annealed that allows an increase in the plastic deformation. The primary creep contribution is recoverable, and it is widely regarded, with some exceptions [13], that the secondary creep contribution is unrecoverable under thermal or irradiation annealing. Based on the Kelly and Foreman model, an elementary irradiation creep model can be constructed as

$\varepsilon=\frac{a \sigma}{E_{0}}[1-\exp (-b \phi)]+k \sigma \phi$

where $\sigma$ is the stress, $E_{0}$ is the Young's modulus of the unirradiated sample, $\phi$ is the fast neutron dose, $a$ and $b$ are constants, and $k$ is defined as the steady state creep coefficient. The first and second terms in Eqn. (2) correspond to the primary and secondary creep, respectively. Experimental data typically indicate that the primary creep saturates with neutron dose at approximately one elastic strain $\left(\sigma / E_{0}\right)$. With this observation, Eqn. (2) can be modified as

$\varepsilon \approx \frac{\sigma}{E_{0}}+k \sigma \phi$

Equations (2) and (3) form the backbone of most creep models that are currently in use; differential forms, in contrast to closed form expressions, are also employed in some analyses [16]. The twocontribution model has been further extended to include structural and radiolytic oxidation changes to Young's modulus for improving the model predictions [13]. A key feature of Eqn. (3) is that the creep is predicted to increase linearly with neutron dose, while experimental data, though with a significant scatter, indicate a significant deviation from linear behavior as shown in Fig. 1.

To account for the non-linear behavior, several models have been developed over the years. The Kelly and Burchell model [17], which was built on the prior work by Kelly and Brocklehurst [9, 18], takes into account the structural changes due to applied stress that manifest as changes in the coefficient of thermal expansion (CTE). The imposition of creep strain alters the CTE of a stressed specimen, increasing it for compressive strain and decreasing it for tensile strain, relative to the unstressed specimens. Thus, Kelly and Burchell model is unique in that it accounts for the sign of the applied stress in predicting the creep strain. The Kelly and Burchell model has been successful in predicting the creep strain for H-451 graphite grade, even at high temperatures, stresses and doses [2] by taking advantage of the correlation between changes in CTE and the creep strain, and the generation of pores at high stresses and temperatures; a more complete discussion is given in Ref. [2]. 

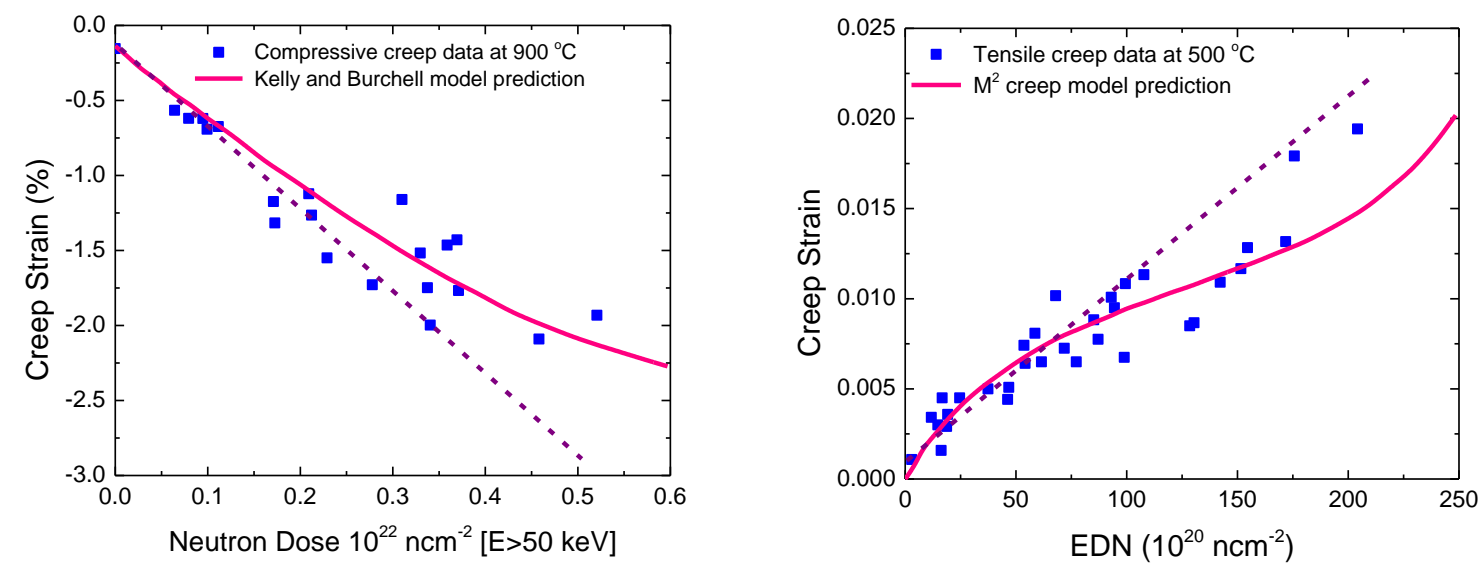

Fig. 1. (left panel): Irradiation creep strain of H-451 graphite specimens at $900{ }^{\circ} \mathrm{C}$ under a compressive stress of 13.8 MPa [8], (right panel): ATR-2E tensile creep data from specimens irradiated at $500{ }^{\circ} \mathrm{C}$ [13]. The prediction from Kelly and Burchell model [17] (left panel), and $\mathbf{M}^{2}$ model (Davies and Bradford, right panel) [13], are shown by continuous red lines. EDN is a dose unit commonly used in the UK based on nickel activation [2].

In Fig. 1 (left panel), the prediction of the Kelly and Burchell model is compared against creep test data for H-451 nuclear graphite grade under compressive stress. Since the change in CTE under a compressive stress is positive and the true creep is negative (which arises from compression loading), the magnitude of the experimental (apparent) creep strain - denoted by the square symbols - will be less than the true creep strain (which varies linearly with neutron dose, and approximated by the broken line in the above figure) [14]. For samples under tensile loading (right panel), the experimental creep strain will again be less than the true creep strain since the change in CTE under a tensile stress is negative along with a positive true strain.

In a recent work, Davies and Bradford [13] have reported a significant recovery of irradiation induced creep at higher fluences along with a diminishing correlation between the CTE and creep. The recovered creep is also shown to be significantly larger than one elastic strain unit $\left(1 e s u \equiv \sigma / E_{0}\right)$. Davies and Bradford have developed a creep model $\left(\mathrm{M}^{2}\right)$ that does not include the effect of CTE explicitly; instead it incorporates a phenomenological term for the recoverable creep. The model has successfully predicted the creep response for several grades of nuclear graphite [13], including H-451. We note here that, while the details differ, both Kelly and Burchell, and $\mathrm{M}^{2}$ models attempt to predict the deviation from the linear behavior at moderate to high neutron doses, as shown in Figure 1. In Kelly and Burchell model, the departure from linear behavior is ascribed to the dimensional changes arising from the stress differential (between stressed and unstressed samples), which is correlated to the changes in the coefficient of thermal expansion, whereas in the $\mathrm{M}^{2}$ model, the deviation from linearity is simply postulated as recoverable creep.

\section{Rate-Theoretic Approach for Irradiation Creep}

While not unusual, the considerable scatter, and hence the uncertainty, in the irradiated creep data precludes a firm validation of the competing models, especially, when they all describe the test data with comparable fidelity. It is observed that irradiation creep is a function of neutron dose, temperature and applied stress, and it depends on the grade of samples that are tested. The differences in the different grades are well-established - nuclear graphite grades are typically polycrystalline materials consisting of either petroleum or pitch-based coke fillers and binder particles. The different constituents and the fabrication procedures have an impact on the microstructure and subsequently affect the irradiation response. Given the lack of clear or unique mechanisms that underpin the irradiation creep behavior, it would be instructive to inquire the dependence of irradiation creep strain on a scalar energy barrier that 
controls the various atomistic and microstructural deformation mechanisms. The creep strain behavior then can be rationalized from the unit processes that closely correlate to the energy barriers. Such an approach is extensively used for describing thermal creep in materials, especially for metals [19-21].

We propose to employ the rate-theoretic approach for analyzing the irradiation creep behavior of graphite. Based on the transition state theory (TST) [22] irradiation creep behavior can be regarded to be an activated process that overcomes a dose-dependent activation energy barrier. Given that the neutron dose is proportional to the time of irradiation, and the general form of creep change with dose is similar to the time-dependent creep behavior we postulate that the irradiation creep rate with time, under the assumption of the harmonic transition theory [22], will have an Arrhenius dependence on the activation energy as shown in Eqn. (4)

$$
\dot{\varepsilon}=\left(\frac{d \varepsilon}{d \phi}\right)\left(\frac{d \phi}{d t}\right) \equiv \dot{\varepsilon}_{\phi}\left(\frac{d \phi}{d t}\right) \propto \exp \left(-\frac{Q_{\phi}}{\hat{R} T}\right)
$$

where $\dot{\varepsilon}_{\phi}$ and $Q_{\phi}$ are the rate of change of irradiation creep with dose $(d \varepsilon / d \phi)$, and activation energy for creep process at the corresponding neutron dose $\phi$, respectively; $T$ is the irradiation temperature in absolute units, and $\hat{R}$ is the universal gas constant. Since neutron dose is proportional to the time of neutron exposure, the time rate of change of neutron dose $(d \phi / d t)$ for any irradiation experiment will be a constant. Thus Eqn. (4) can be rewritten as

$$
\dot{\varepsilon}_{\phi}=A \exp \left(-\frac{Q_{\phi}}{\hat{R} T}\right)
$$

where $A$ is a constant. It may be noted here that Eqn. (5) is completely analogous to activated thermal creep expression, except that the change in creep with time is replaced by the change in creep with neutron dose. The applied stress $(\sigma)$ provides the driving force for plastic flow, which is controlled by dislocation mobility and diffusion of defects. As the stress is increased, the rate of deformation also increases, though not always, linearly. In general, it is observed that the strain rate has a power-law dependence on the applied stress for thermal creep [23]. We will assume a similar power-law for irradiation creep and will postulate that

$$
\dot{\varepsilon}_{\phi} \propto \sigma^{n}
$$

where $n$ is termed as the stress exponent and its value depends on the specific mechanism of creep such as lattice diffusion or dislocation motion. Combining Eqns. (5) and (6), the governing equation for the creep strain rate can be expressed as [23, 24]:

$$
\dot{\varepsilon}_{\phi}=C \sigma^{n} \exp \left(-\frac{Q_{\phi}}{\hat{R} T}\right)
$$

where $C$ is a constant of proportionality. Equation (7) now can be rearranged into the form:

$$
\ln \dot{\varepsilon}_{\phi}=\ln C+n \ln \sigma-\frac{Q_{\phi}}{\hat{R} T}
$$

Thus, as shown in Figure 2 (left panel), the activation energy for any neutron dose $Q_{\phi}$ can be determined experimentally, by plotting the natural logarithm of the creep rate with dose $\left(\dot{\varepsilon}_{\phi}\right)$ against the reciprocal of temperature, while the stress exponent $n$ can be determined by plotting the strain rate as a function of 
stress in a log-log scale as shown in Figure 2 (right panel). With statistically significant test data at different temperatures and stresses, the activation energy and stress exponent can be evaluated with reasonable confidence. It may be further noted here that the numerical value of the constant $C$ does not influence the stress exponent or the activation energy, which indicates that these variables are not dependent on the neutron flux $\left(n . \mathrm{cm}^{-2} \cdot \mathrm{s}^{-1}\right)$ at the test location. Thus samples of the same grade from different reactors and test conditions can be analyzed consistently.
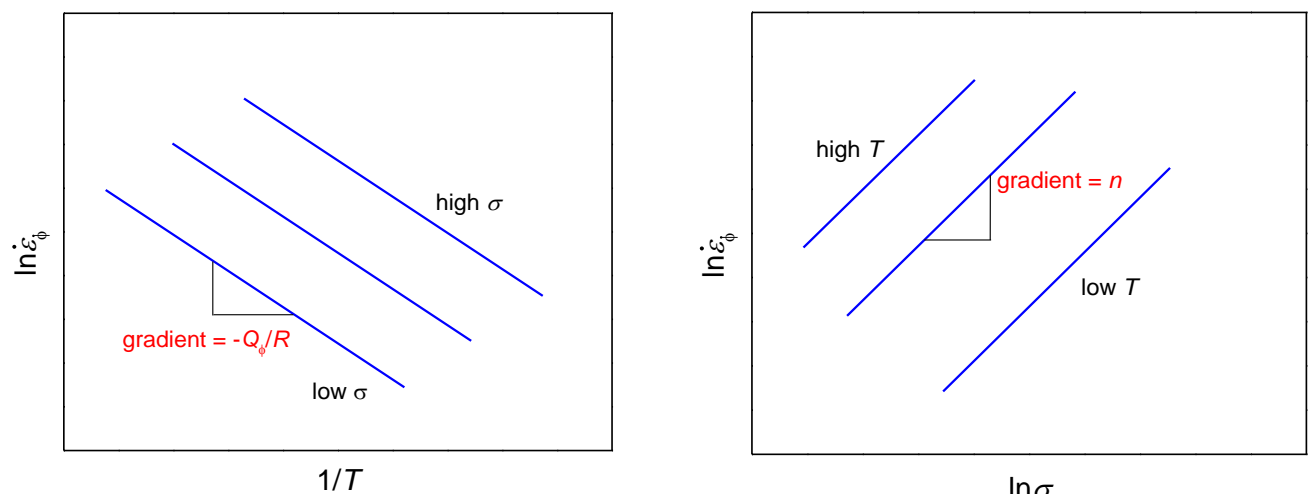

Fig. 2 Schematic variation of logarithm of creep rate with dose $\left(\ln \dot{\varepsilon}_{\phi}\right)$ with $\ln \sigma$ verse temperature $(1 / T)$ to determine activation energy $\left(Q_{\phi}\right)$ (left panel), and with logarithm of applied stress $(\ln \sigma)$ to determine the stress exponent $(n)$ (right panel).
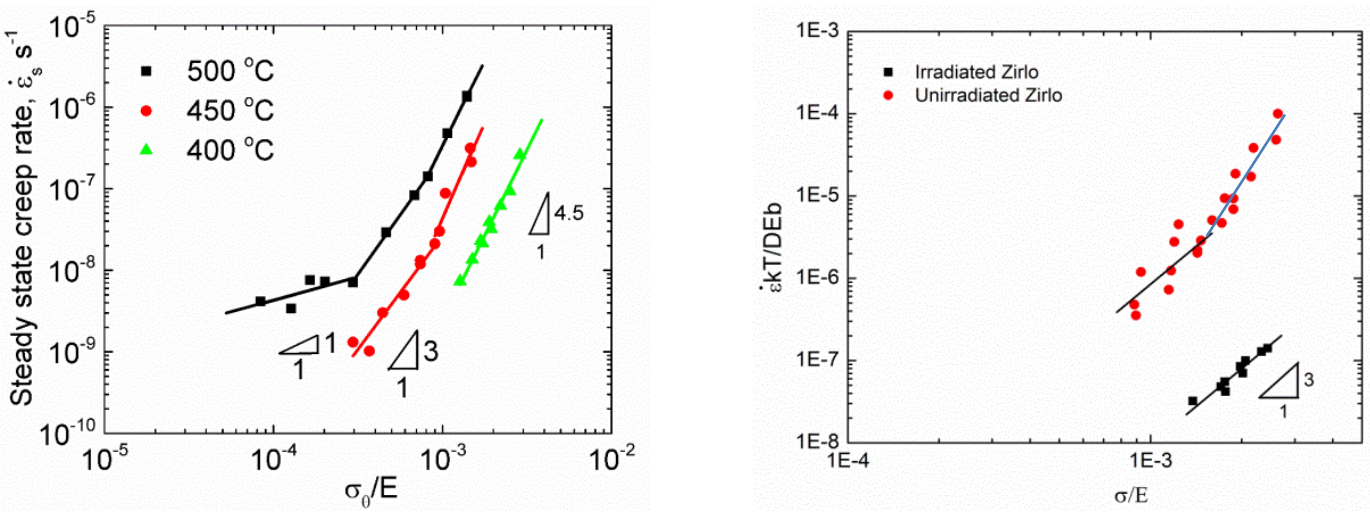

Fig. 3 (left panel) Steady state creep in a zirconium alloy (HANA-4 ${ }^{\mathrm{TM}}$ ) under different temperatures, and (right panel) normalized creep strain rate as a function of normalized stress in virgin and irradiated Zirlo $^{\mathrm{TM}}[25]$.

We will first discuss how thermal creep is traditionally analyzed in the rate-theoretic framework, and how it can be extended for irradiation induced creep. In general, three stages have been identified for thermal creep of metals [26]. In the initial or primary creep stage, the strain rate is relatively high, but slows with increasing time due to work hardening. The strain rate eventually reaches a minimum and becomes nearly constant due to the balance between work hardening and annealing (thermal softening) resulting in the secondary or steady-state creep regime. This stage is well-understood and the reported creep strain rates in metals typically refer to the minimum rate in the secondary stage. Stress dependence of this rate depends on the underlying creep deformation mechanism. In the tertiary creep regime, the strain rate increases exponentially with stress leading to fracture, eventually. We will briefly discuss how the thermal and irradiation creep mechanisms can be identified in Zirconium alloys, a clad material for current Light Water Reactors (LWRs) from the rate theory perspective as shown in Fig. (3). 
The left panel of Fig. (3) delineates steady state (secondary) creep rate in HANA-4 ${ }^{\mathrm{TM}}-$ a zirconium alloy developed in Korea [25] - for different normalized stresses at various temperatures. As common with other metals, the creep strain rate shows several regimes with different stress exponents. At low stress levels, the stress exponent varies between 1 and 2 [27] with relatively low creep strain rates. While a stress exponent of 2 is commonly attributed to grain boundary sliding, several mechanisms can contribute to the so-called viscous creep with $n=1$. Bulk diffusional transport through the lattice (Nabarro-Herring) and grain boundary diffusional (Coble) mechanisms are commonly noticed in the viscous creep regime; dislocation motion and migration of vacancies can also result in viscous creep as in Harper-Dorn creep mechanism [27, 28]. At higher stresses, the stress exponent changes to 3, approximately, followed by an even higher stress exponent. A stress exponent of 3 typically indicates a deformation mechanism through dislocation glide in metals; higher values of stress exponent indicate a crossover to a dislocation climb and cross-slip mechanisms [20].

The right panel of Figure (3) depicts the normalized creep strain rate as a function of normalized stress in virgin and irradiated Zirlo ${ }^{\mathrm{TM}}$ - a zirconium alloy developed at Westinghouse in the Unites States [29]. Interestingly after irradiation, while there is almost two-orders reduction in the normalized strain rate for the same stress state, the stress exponent remarkably remains constant at 3, approximately, at moderate stress levels. The invariant stress exponent has two imports. A well-identifiable power-law exponent shows that the irradiation induced creep follows activated dynamics, which confirms the applicability of the transition state rate theory formalism after irradiation. The second implication is that the creep mechanism - namely, dislocation glide - is operational for the irradiated samples as well. Though data is scarce, similar behavior has been obtained in other irradiated materials as well. We also note here that Karthik et al. [30] recently found evidence for the formation of dislocation dipoles from vacancy loops in NBG-18, a newer nuclear grade graphite [31], under electron irradiation. Using in-situ measurements with HRTEM, the formation of a vacancy loop is observed first, followed by a dissociation into a set of dislocations and the formation of incomplete planes through a climb mechanism.

In the modified theory for irradiation creep, as discussed before, the time rate of creep is replaced by dose rate of creep. Thus, analogously, different creep mechanisms can be ascertained by extracting the activation energy $\left(Q_{\phi}\right)$ and the stress exponent $(n)$. As with thermal creep, the analysis is limited to the conditions where the creep change with dose is sensibly constant, which is generally referred to as the secondary or steady-state irradiation creep regime. The primary objective of this formalism is to uncover the effects of temperature and stress assuming that the steady-state irradiation creep is well-described by activated dynamics and harmonic transition state rate theory.

\section{Analysis of irradiation creep data using rate theory formalism}

4.1 Irradiation Creep and Creep Rate with Dose: While irradiation creep data for different grades of graphite are available in literature, most of the data are for limited stress and temperature conditions. As noted in Section 3, data for a particular grade at different stresses and temperatures are required to analyze the creep data in the rate theory framework to determine the optimal values of $n$ and $Q$. Given the paucity of data, we limit our analysis to two grades of graphite H-337 and AGOT [7], for which a reasonable inference can be made on the stress exponent and the activation energy barrier; these grades are particularly chosen because they have been tested at the same stresses $(6.9,13.8$ and $20.7 \mathrm{MPa})$ and temperatures $\left(550{ }^{\circ} \mathrm{C}\right.$ and $800{ }^{\circ} \mathrm{C}$ ), and in the same reactor. The microstructural details of $\mathrm{H}-337$ and AGOT grades are provided in Table 1 with the data derived from Ref. [32] and Ref. [33], respectively. The creep data used for the current analysis is taken from Ref. [7] for both H-337 and AGOT grades . 
Table 1 Microstructural details and thermo-mechanical properties of H-337 and AGOT nuclear graphite grades [32, 33].

\begin{tabular}{|c|c|c|c|c|c|c|}
\hline $\begin{array}{c}\text { Graphite } \\
\text { Grade }\end{array}$ & $\begin{array}{c}\text { Method } \\
\text { of } \\
\text { Forming }\end{array}$ & $\begin{array}{c}\text { Filler } \\
\text { Description }\end{array}$ & Binder & Density & $\begin{array}{c}\text { CTE } \times 10^{6} \\
/{ }^{\circ} \mathrm{C}\end{array}$ & $\begin{array}{c}\text { Young's } \\
\text { Modulus } \\
\times 10^{-6}(\mathrm{psi})\end{array}$ \\
\hline H-337 & Molded & $\begin{array}{c}\text { Medium } \\
\text { semiacicular }\end{array}$ & Binderless & 1.96 & 5.1 & 2.07 \\
\hline AGOT & Extrusion & $\begin{array}{c}\text { Coarse } \\
\text { acicular }\end{array}$ & $\begin{array}{c}\text { Coal-tar } \\
\text { pitch }\end{array}$ & 1.71 & 1.3 & 1.77 \\
\hline
\end{tabular}
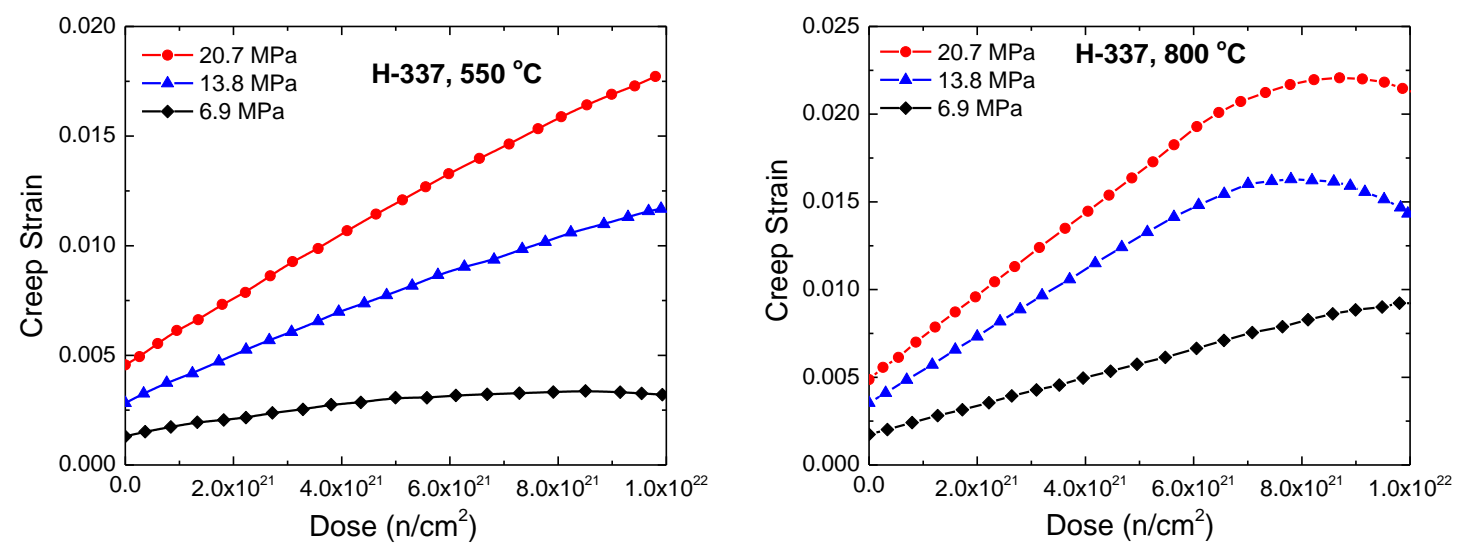

Fig. 4 Creep strain against dose for $\mathrm{H}-337$ at $550{ }^{\circ} \mathrm{C}$ (left panel), and at $800{ }^{\circ} \mathrm{C}$ (right panel). The data has been taken from Ref. [7].
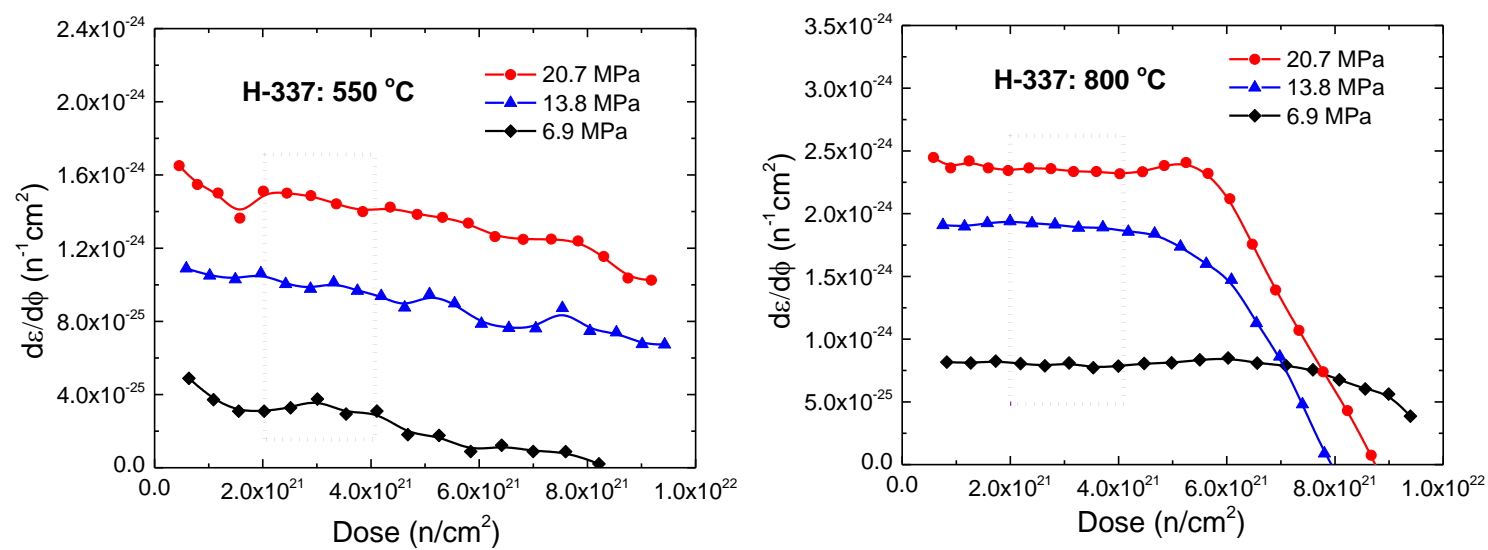

Fig. 5 Creep rate with dose for $\mathrm{H}-337$ samples at $550{ }^{\circ} \mathrm{C}$ (left panel), and $800{ }^{\circ} \mathrm{C}$ (right panel). The rate of creep with dose is approximately constant in the range shown by the boxes at both temperatures.

Figure 4 shows the creep strain for $\mathrm{H}-337$ as a function of dose for two temperatures $550{ }^{\circ} \mathrm{C}$ (left panel) and $800{ }^{\circ} \mathrm{C}$ (right panel) while Figure 5 shows the corresponding rate of change of strain with dose for the two temperatures; the rate of creep with dose is approximately constant in the range shown by the boxes at both temperatures. The activated dynamics approach is most appropriate when the rate of change of strain remains steady when comparing between different temperatures 
and stresses. From Figs. 4 and 5, the rate can be treated as approximately constant for doses between $2 \times 10^{21}$ and $4 \times 10^{21} \mathrm{n} / \mathrm{cm}^{2}$. Figure 6 delineates the irradiation creep data for AGOT grade graphite samples. It can be observed that the creep data for AGOT is qualitatively similar to that in H-337 (see Fig. 4). As the stresses increase, the creep also increases although irradiation creep recovery is pronounced at $800{ }^{\circ} \mathrm{C}$ in grade $\mathrm{H}-337$ for 20.7 and $13.8 \mathrm{MPa}$ stress levels. This turnaround behavior in the creep data is reflected as noticeable variation in the creep rate (see right panel in Fig. 5). For AGOT, creep recovery is marginal though there is a creep saturation behavior at $550{ }^{\circ} \mathrm{C}$ and $20.7 \mathrm{MPa}$, which is accompanied by a sharper gradient in the creep rate (see Fig. 7). Overall, the change in creep is approximately linear for both samples at low doses. For the current analysis, therefore, we will use the creep strain at $4 \times 10^{21} \mathrm{n} / \mathrm{cm}^{2}$, which is a representative value for low neutron dose. By choosing the nearly steady-state creep rates, we can compare the activation energies and stress exponents consistently between the samples as well between different creep test conditions. It may be noted here that qualitative changes in the activated dynamics can be evaluated at other doses even though care must be taken when interpreting the data.
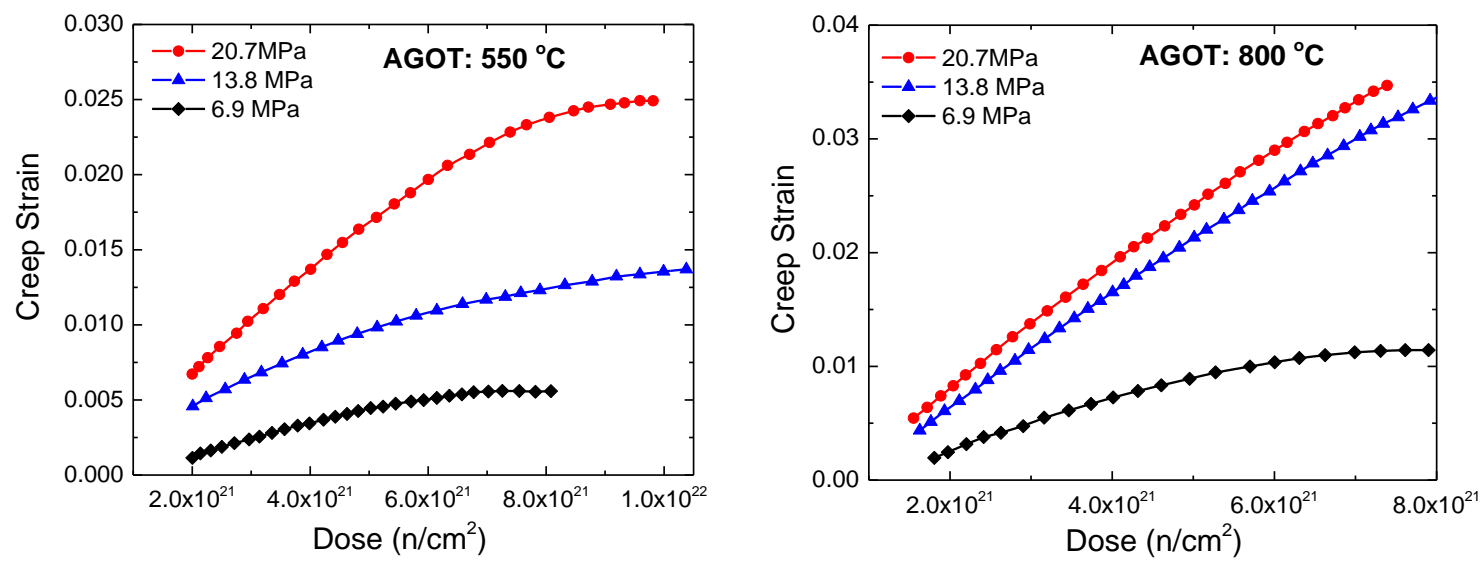

Fig. 6 Creep strain against dose for AGOT graphite at $550{ }^{\circ} \mathrm{C}$ (left panel) and $800{ }^{\circ} \mathrm{C}$ (right panel). The data has been taken from Ref. [7].
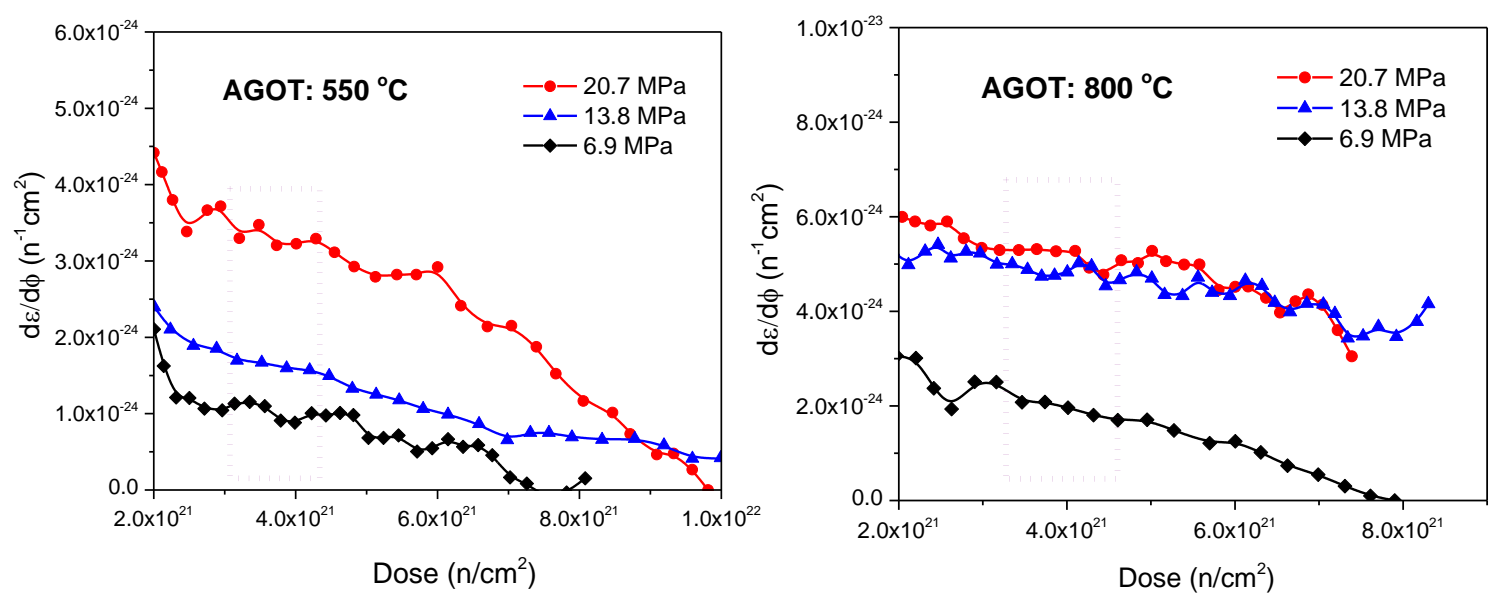

Fig. 7 Creep rate with dose for AGOT samples at $550{ }^{\circ} \mathrm{C}$ (left panel), and $800{ }^{\circ} \mathrm{C}$ (right panel). The rate of creep with dose is approximately constant in the range shown by the boxes at both temperatures. 
4.2 Stress Exponents: We will first present the stress exponents as evaluated from Eqn. (8) and Figure 2 (right panel). Figure 8 depicts the rate of change of strain with dose for H-337 and AGOT samples for different temperatures, as a function of stress. Several interesting features can be gleaned from the creep rate data. First, there is an identifiable stress exponent for both H-337 and AGOT samples as evidenced by the three creep rate data points falling in a line for each temperature. This power law behavior indicates that the irradiation creep data indeed can be analyzed within the activated dynamics framework at low doses. The second noticeable characteristic is that the mean stress exponent of 1.02 for all data sets with a maximum deviation of \pm 0.19 . A stress exponent of unity indicates that the strain rate (with dose) is proportional to stress, which is typically identified as viscous creep. In metals, a stress exponent of unity usually exemplifies a diffusional mechanism for deformation rather than influenced by dislocation motion that typically have larger values $(\geq 3)$. A firmer picture on the creep mechanism, though with extremely limited data that is generally a characteristic of irradiation creep analysis, can be constructed by analyzing the activation energy.
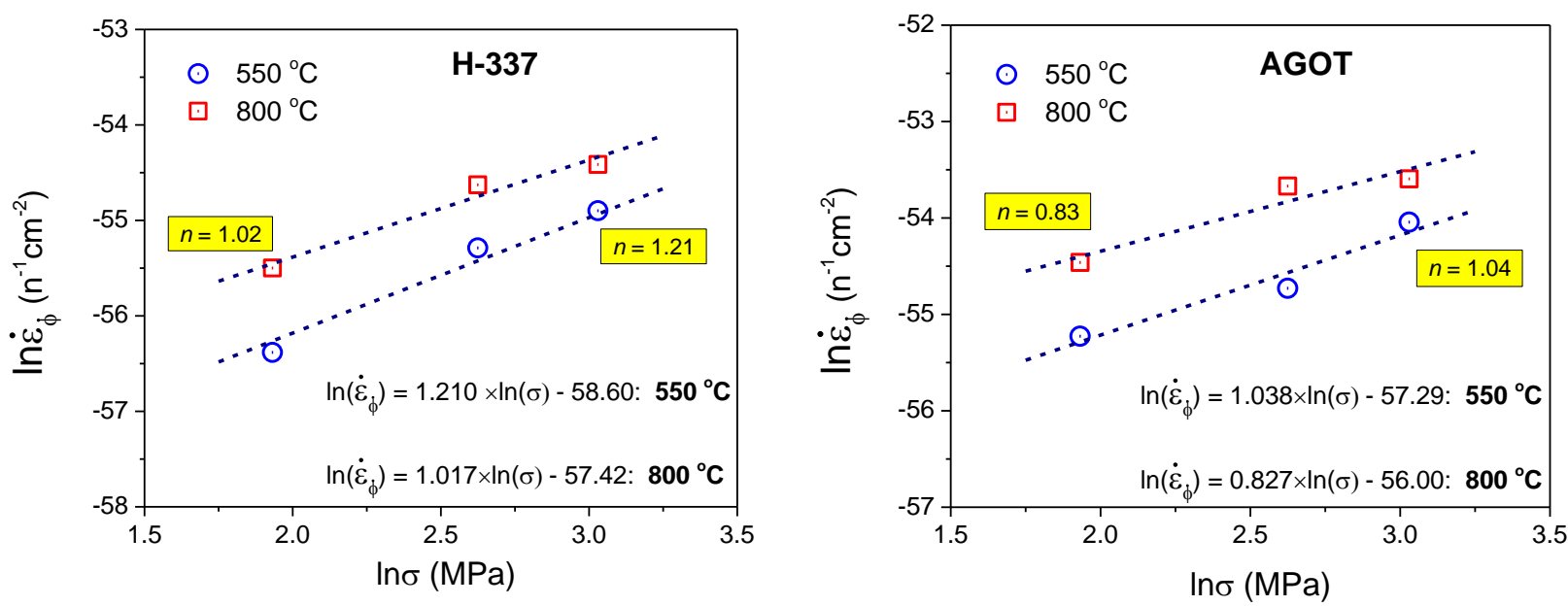

Fig. 8 Stress exponents for H-337 (left panel) and AGOT (right panel) samples. For H-337 and AGOT samples for both temperatures, the stress exponents are nearly equal to 1 with a maximum deviation of \pm 0.2 , suggesting a viscous creep mechanism for low neutron doses (in the range of 3 to $4 \times 10^{22} \mathrm{n} / \mathrm{cm}^{2}$ ).

\subsection{Activation Energy:}

Table 2 Activation energy at different stresses for H-337 and AGOT samples

\begin{tabular}{|c|c|c|}
\hline \multirow{2}{*}{ Stress (MPa) } & \multicolumn{2}{|c|}{ Activation Energy (eV) } \\
\cline { 2 - 3 } & H-337 & AGOT \\
\hline 6.9 & 0.27 & 0.23 \\
\hline 13.8 & 0.20 & 0.32 \\
\hline 20.7 & 0.15 & 0.14 \\
\hline
\end{tabular}

Since data is available only for two temperatures, the activation energy can be evaluated with only two creep dose rates. As shown in Table 1, the activation energy varies between 0.32 to $0.14 \mathrm{eV}$ for different stress states. With increasing stress, the activation energy decreases, which is consistent with the 
transition state theory [22]. The activation energy is a measure of the change in the Gibbs free energy $(d G)$ and in typical creep experiments with the specimens held at constant normal stress $(\sigma)$ and temperature $(T), d G$ can be expressed as

$d G=d U+\sigma d V-T d S$

where $d U$ is the change in internal or potential energy, $d V$ is the volume change, $d S$ is the entropy change, and $\sigma$ is the applied stress; the sum of the first two terms is the change in enthalpy at constant stress. Thus, in general, the activation energy at any neutron dose is both a function of temperature and stress, which is shown as: $Q_{\phi}=Q_{\phi}(\sigma, T)$. Creep experiments in alloys typically show that activation energy decreases with increasing stress [34]; for example, the activation energy in copper decreases by $60 \%$, approximately, when stress is increased 3 times from (50 to $150 \mathrm{MPa}$ ); in engineering alloys, a weak to moderate stress dependence is typically observed.

From Table 1, except for one data point (AGOT - 13.9 MPa), the activation energy appears to decrease with increasing stress, which is consistent with data extracted from typical thermal creep experiments. It is also interesting to note that the activation energy is similar for both H-337 and AGOT at the same stresses (with one exception) indicating that the creep mechanism in both grades is similar. There is one important difference in the activation energy for graphite grades relative to metallic alloys. In the viscous regime, the activation energies are typically of the order of $1 \mathrm{eV}$; for example, HANA 4 alloy exhibits a Coble creep mechanism with a mean activation energy of $1 \mathrm{eV}$, approximately. In the two graphite grades, however, the activation energies are significantly smaller ( 0.15 to $0.3 \mathrm{eV})$.

As discussed before, the stress exponent of unity at all temperatures indicates a diffusion mechanism. We will now discuss the various defects types that are commonly observed in graphite under irradiation. Graphite has a lamellar structure, which is generated by several stacks of basal planes. Each basal (graphene) plane is constituted by strong, $s p^{2}$-hybridized carbon atoms arranged in a hexagonal lattice. The weak interlayer interaction allows the graphitic layers to slip over each other promoting the formation of complex interlayer defects $[35,36]$. Irradiation typically creates a large number of point defects, a fraction of which will condense over time to form extended defects. It is known that several types of point defects (see Fig. 9) are energetically possible, many of them involving closed planar structures $\left(s p^{2}\right.$ hybridized) or three dimensional structures ( $s p^{3}$-hybridized, or a combination of $s p^{2}$ and $\left.s p^{3}\right)$ [36-39]. A monovacancy can be created by removing an atom from the basal plane, which renders a Jahn-Teller distortion to the basal plane with two atoms closest to the monovacancy moving further closer to form a pentagon-like structure, and the third closest atom protruding out of the plane [40]; the distortion is manifested prominently for monovacancies on a surface. By removing two adjacent atoms from the basal plane a divacancy can be formed, which upon relaxation forms a 5-8-5 topological defect [41]. Both experiments and electronic structure simulations predict nearly similar formation energies for mono and divacancies. For diffusional transport, electronic structure simulations predict a migration energy of 1.1 $\mathrm{eV}$ for a monvacancy [42], and $2.17 \mathrm{eV}$ for a divacancy [40], both of which are much higher than the activation energy extracted from the current experiments. Experimental migration energy for monovacancies is $3 \mathrm{eV}$, approximately; however, this value is deemed too high as it is suspected that the experiments reveal an average migration energy of several types of vacancy defects. Since the migration energies fall in the range of 1 to $3 \mathrm{eV}$, we conclude that the diffusional mechanism observed in the current work does not arise from diffusional transport of mono or divacancies. 

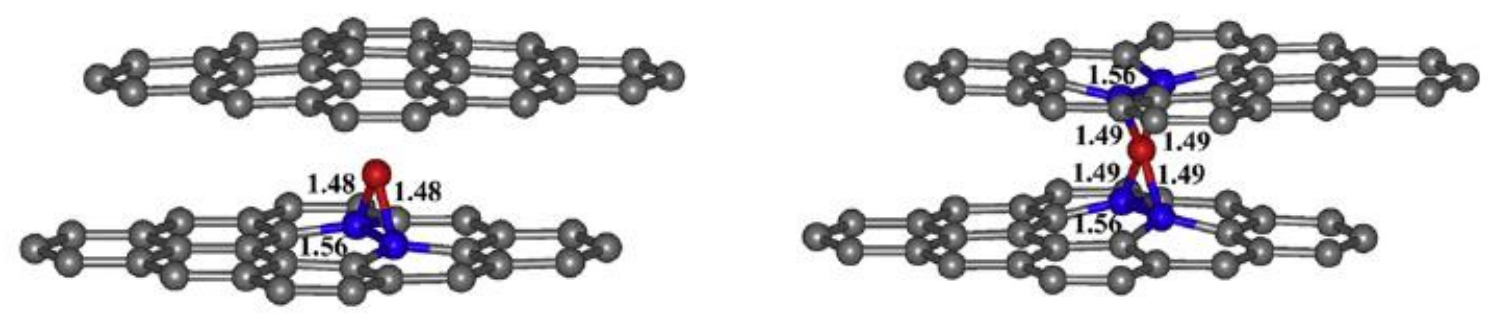

Fig. 9 Bridge (left panel) and spiro (right panel) defect configuration [40]. The red atoms depict interstitial atoms and the numbers indicate bond distances in $\AA$. The migration energy for Spiro configuration is $2.12 \mathrm{eV}$, while that for the bridge configuration is $0.36 \mathrm{eV}$ [40].

In-plane interstitials do not exist in graphite as the energy penalty for placing an atom in the hexagonal ring is extraordinarily high. Telling and co-workers [36] have shown that vacancy and interstitial defects can form complex interplanar defects such as spiro, bridge and ylid, which are energetically favorable [43]. Of particular interest is the effect of low threshold for shearing the basal planes [36] - on account of the weak interlayer bonding energy, which allows interstitial atoms to bond between the basal layers. Such formations are essentially three dimensional and include non- $s p^{2}$ bonded structures (see Fig. 9). Using electronic structure simulations, Zhang and co-workers have estimated the migration energies for the interstitial configurations and have obtained $2.12 \mathrm{eV}$ and $0.36 \mathrm{eV}$ for the spiro and bridge configurations, respectively. The migration energy of the bridge configuration is the closest to what we have obtained from the creep experiments $(0.15-0.32 \mathrm{eV})$. However, as discussed before graphite accommodates basal dislocations too; in the widely accepted Kelly and Foreman model the primary plastic deformation mechanism is through the flow of basal dislocations. Small interstitial clusters that are formed between the basal planes act as pinning sites, which impede the motion of basal dislocations. Basal dislocations, generally, do not break the strong in-plane atomic bonds, rather they are created by distorted carbon-carbon bonds on the basal plane. First principles simulations show that the Peierls stress barrier for basal dislocation glide is quite small $\left(10^{-17} \mathrm{~Pa}\right)$; the shear strength, therefore, is largely dependent on the pinning of dislocations [44]. While the small stress barriers correspond to activation energies that are of the order of $\mathrm{meV}$ [36], pinning of the basal dislocations can significantly increase the activation energy barrier.

\subsection{Molecular Dynamics Simulations}

To investigate the possibility of bridge-like defect formations under irradiation, we have conducted displacement cascade molecular dynamics (MD) simulations on graphite. The main objective is to examine the probability of generating bride-like defects during a displacement cascade process. Using the AIREBO interatomic potential [45], that has been benchmarked to a large number of experimental and electronic structure first principles simulations [46], we perturb a cubic system of 500,000 atoms, approximately, by imparting excess energy and momentum to a primary knock-on atom (PKA). Knock directions both along and perpendicular to the basal planes have been considered with knock energies ranging from 1 to $7 \mathrm{keVs}$. To improve the statistics of defect formations, 50 independent configurations with different initial positions and momenta have been used; more details will be presented elsewhere [47]. 


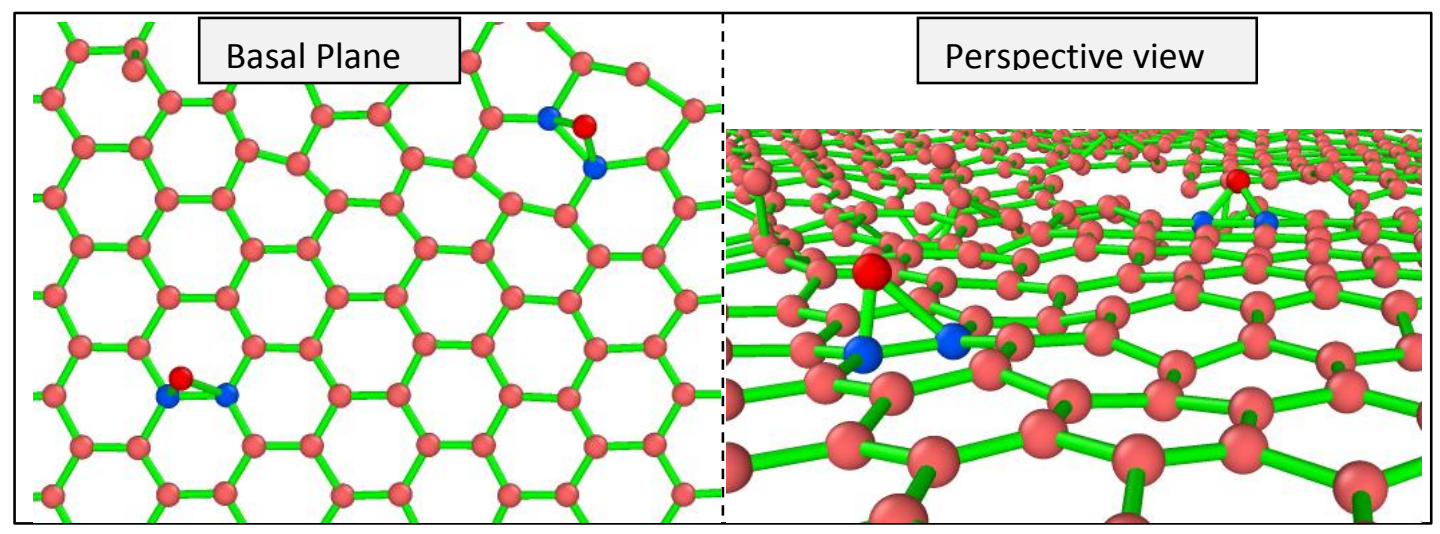

Fig. 10 Bridge-like defect configurations observed during the early stages of defect evolution (0.03 ps after initiating the knock)

As depicted in Fig. 10, our simulations show the formation of the bridge-like configurations in the early stages of the defect evolution. However, they are unstable and with continued energy dissipation, the interstitials migrate and form aggregates. Thus, as shown in Fig. 11 (left panel), the bridge-like defect configurations quickly decrease. Using energy minimized structures after 10 ps we do not find any evidence for bridge or spiro configurations. Instead the defect structures are largely comprised of vacancy/interstitial aggregates; Fig. 11 (right panel) shows two such configurations, which together comprise over $40 \%$ of the total point defects. We also note that our results are consistent with an electronic first principles investigation, and recent MD simulations [48, 49] simulating radiation damage, which noted the absence of bridge, spiro and ylid structures in irradiated graphite [50].
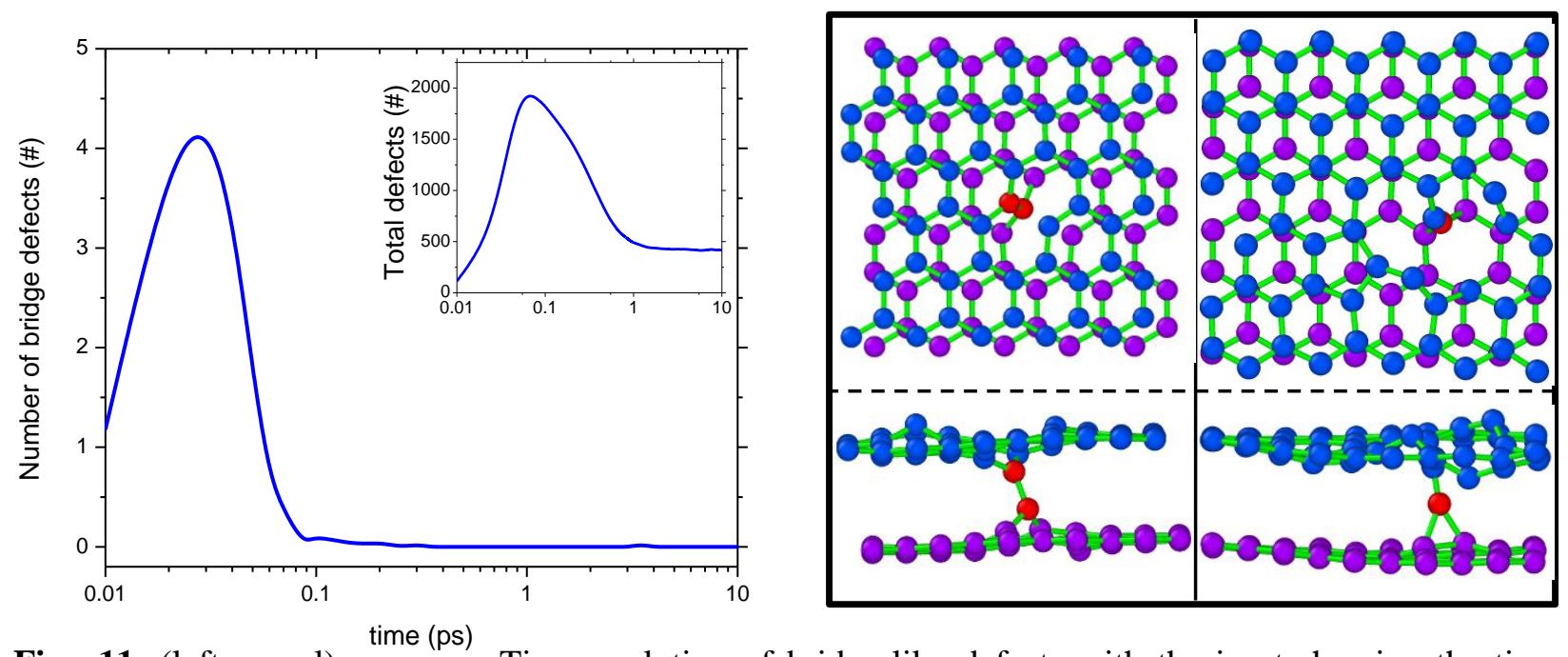

Fig. 11 (left panel) Time evolution of bridge-like defects with the inset showing the time variation of the total number of defects for a $7 \mathrm{keV}$ knock along the basal plane (averaged over 50 runs). (right panel) Statistically significant interstitial/vacancy configurations; color coding: blue $\rightarrow$ top layer, purple $\rightarrow$ bottom layer, red $\rightarrow$ interstitial. Similar results are obtained with a knock perpendicular to the basal plane.

Our MD simulations thus indicate that bridge-like defects are unlikely to be present in significant numbers in irradiated graphite. Given the large energy barriers for the interlayer defects, which are of the order of $1 \mathrm{eV}$, the irradiation creep deformation process is likely to be mediated through the flow of basal dislocations, as originally proposed by Kelly and co-workers. 


\section{Concluding Remarks}

In summary, we have examined irradiation induced creep of graphite in the framework of transition state rate theory. Experimental data for two grades of nuclear graphite (H-337 and AGOT) have been analyzed to determine the stress exponent $(n)$ and activation energy $(Q)$ for plastic flow under irradiation. We show that the mean activation energies $(Q)$ lie between $0.15-0.32 \mathrm{eV}$ with a mean stress-exponent of $1.02 \pm 0.19$. Theoretical and computational analyses favor an irradiation creep deformation mechanism that is mediated through the flow of basal dislocations, which is consistent with the creep models of graphite. Given the paucity of irradiation creep data at different stresses and temperatures, more experimental work and analyses are suggested to arrive at a more definitive conclusion.

Acknowledgements: The authors gratefully acknowledge the financial support from the Nuclear Energy University Program (NEUP) of Department of Energy (DoE) for performing this research.

\section{References}

[1] B.T. Kelly, B.J. Marsden, K. Hall, D.G. Martin, A. Harper, A. Blanchard, J. Kendall, Irradiation Damage in Graphite due to Fast Neutrons in Fission and Fusion Systems, IAEA - TECDOC 1154, (2000).

[2] T.D. Burchell, K.L. Murty, J. Eapen, Irradiation induced creep of graphite, JOM, 62 (2010) 9399.

[3] D.G. Martin, R.W. Henson, The effect of irradiation temperature on the scattering of long wavelength neutrons by defects in neutron-irradiated graphite, Carbon, 5 (1967) 313.

[4] B.T. Kelly, A.J.E. Foreman, The theory of irradiation creep in reactor graphite-The dislocation pinning-unpinning model, Carbon, 12 (1974) 151-158.

[5] B.T. Kelly, J.E. Brocklehurst, UKAEA Reactor Group studies of irradiation-induced creep in graphite, Journal of Nuclear Materials, 65 (1977) 79-85.

[6] G.M. Jenkins, D.R. Stephen, The temperature dependence of the irradiation induced creep of graphite, Carbon, 4 (1966) 67-72.

[7] W.J. Gray, Constant stress irradiation-induced compressive creep of graphite at high fluences, Carbon, 11 (1973) 383-392.

[8] T.D. Burchell, Irradiation induced creep behavior of H-451 graphite, Journal of Nuclear Materials, 381 (2008) 46-54.

[9] J.E. Brocklehurst, B.T. Kelly, Analysis of the dimensional changes and structural changes in polycrystalline graphite under fast neutron irradiation, Carbon, 31 (1993) 155-178.

[10] J.E. Brocklehurst, R.G. Brown, Constant stress irradiation creep experiments on graphite in BR-2, Carbon, 7 (1969) 487-497.

[11] R. Blackstone, Radiation creep of graphite. An introduction, Journal of Nuclear Materials, 65 (1977) 72-78.

[12] X. Fang, H. Wang, S. Yu, C. Li, The various creep models for irradiation behavior of nuclear graphite, Nuclear Engineering and Design, 242 (2012) 19-25.

[13] M.A. Davies, M. Bradford, A revised description of graphite irradiation induced creep, Journal of Nuclear Materials, 381 (2008) 39-45.

[14] T.D. Burchell, Irradiation induced creep behavior of H-451 graphite, Journal of Nuclear Materials, 381 (2008) 46-54.

[15] B.T. Kelly, A.J.E. Foreman, "The Theory of Irradiation Creep in Reactor Graphite - The Dislocation Pinning-Unpinning Model, Carbon, 12 (1974) 151.

[16] C. Erasmus, S. Kok, M.P. Hindley, Significance of primary irradiation creep in graphite, Journal of Nuclear Materials, 436 (2013) 167-174.

[17] B.T. Kelly, T.D. Burchell, Structure-related property changes in polycrystalline graphite under neutron irradiation, Carbon, 32 (1994) 499-505. 
[18] B.T. Kelly, Irradiation creep in graphite - some new considerations and observations, Carbon, 30 (1992) 379-383.

[19] F.R.N. Nabarro, F.d. Villiers, Physics Of Creep And Creep-Resistant Alloys, Taylor and Francis, London, 1995.

[20] B. Kombaiah, K.L. Murty, Dislocation cross-slip controlled creep in Zircaloy-4 at high stresses, Materials Science and Engineering: A, 623 (2015) 114-123.

[21] A. Sarkar, K. Boopathy, J. Eapen, K.L. Murty, Creep Behavior of Hydrogenated Zirconium Alloys, J. of Materi Eng and Perform, 23 (2014) 3649-3656.

[22] A. Kushima, J. Eapen, J. Li, S. Yip, T. Zhu, Time scale bridging in atomistic simulation of slow dynamics: viscous relaxation and defect activation, Eur. Phys. J. B, 82 (2011) 271-293.

[23] G.E. Dieter, Mechanical metallurgy, McGraw-Hill New York, 1976.

[24] I. Charit, K.L. Murty, Creep behavior of niobium-modified zirconium alloys, Journal of Nuclear Materials, 374 (2008) 354-363.

[25] B. Kombaiah, K.L. Murty, Coble, Orowan Strengthening and Dislocation Climb mechanisms in a Nb-modified Zircaloy cladding, Met Mat Trans, 46 (2015) 4646-4660.

[26] M.E. Kassner, Fundamentals of Creep in Metals and Alloys, II ed., Elsevier Oxford, UK, 2009.

[27] F.R.N. Nabarro, Creep at very low rates, Metallurgical and Materials Transactions A, 33 (2002) 213-218.

[28] P. Kumar, M. Kassner, T. Langdon, Fifty years of Harper-Dorn creep: a viable creep mechanism or a Californian artifact?, Journal of Materials Science, 42 (2007) 409-420.

[29] G. Wikmark, Testing and Modeling of Long-term Creep of Modern PWR Cladding in Dry Storage and Transport, , in: Water Reactor Fuel Performance Meeting, Chengdu, China, (Sept. 11-14), 2011.

[30] C. Karthik, J. Kane, D.P. Butt, W.E. Windes, R. Ubic, In situ transmission electron microscopy of electron-beam induced damage process in nuclear grade graphite, Journal of Nuclear Materials, 412 (2011) 321-326.

[31] C. Karthik, J. Kane, D.P. Butt, W.E. Windes, R. Ubic, Microstructural Characterization of Next Generation Nuclear Graphites, Microscopy and Microanalysis, 18 (2012) 272-278.

[32] C.R. Kennedy, Irradiation of graphite at $950^{\circ} \mathrm{C}$, Techinical Report 197322, ORNL, (1973).

[33] W.L. Greenstreet, J.E. Smith, G.T. Yahr, Mechanical properties of EGCR-type AGOT graphite, Carbon, 7 (1969) 15-45.

[34] A.D. Freed, S.V. Raj, K.P. Walker, Stress Versus Temperature Dependence of Activation Energies for Creep, Journal of Engineering Materials and Technology, 114 (1992) 46-50.

[35] R.H. Telling, M.I. Heggie, Radiation defects in graphite, Philosophical Magazine, 87 (2007) 4797-4846.

[36] R.H. Telling, C.P. Ewels, A.A. El-Barbary, M.I. Heggie, Wigner defects bridge the graphite gap, Nat Mater, 2 (2003) 333-337.

[37] F. Banhart, J. Kotakoski, A.V. Krasheninnikov, Structural Defects in Graphene, ACS Nano, 5 (2010) 26-41.

[38] H. Terrones, R. Lv, M. Terrones, M.S. Dresselhaus, The role of defects and doping in 2D graphene sheets and 1D nanoribbons Rep. Prog. Phys., 75 (2012) 062501.

[39] J. Kotakoski, A.V. Krasheninnikov, Chapter 11 Native and Irradiation-Induced Defects in Graphene: What Can We Learn from Atomistic Simulations?, in: Computational Nanoscience, The Royal Society of Chemistry, 2011, pp. 334-376.

[40] H. Zhang, M. Zhao, X. Yang, H. Xia, X. Liu, Y. Xia, Diffusion and coalescence of vacancies and interstitials in graphite: A first-principles study, Diamond and Related Materials, 19 (2010) 12401244.

[41] J. Eapen, R. Krishna, T.D. Burchell, K.L. Murty, Early Damage Mechanisms in Nuclear Grade Graphite under Irradiation, Materials Research Letters, 2 (2013) 43-50. 
[42] C.D. Latham, M.I. Heggie, M. Alatalo, S. Öberg, P.R. Briddon, The contribution made by lattice vacancies to the Wigner effect in radiation-damaged graphite, Journal of Physics: Condensed Matter, 25 (2013) 135403.

[43] L. Li, S. Reich, J. Robertson, Defect energies of graphite: Density-functional calculations, Physical Review B, 72 (2005) 184109.

[44] R.H. Telling, M.I. Heggie, Stacking fault and dislocation glide on the basal plane of graphite, Philosophical Magazine Letters, 83 (2003) 411-421.

[45] S.J. Stuart, A.B. Tutein, J.A. Harrison, A reactive potential for hydrocarbons with intermolecular interactions, The Journal of Chemical Physics, 112 (2000) 6472-6486.

[46] C.D. Latham, A.J. McKenna, T.P. Trevethan, M.I. Heggie, M.J. Rayson, P.R. Briddon, On the validity of empirical potentials for simulating radiation damage in graphite: a benchmark, Journal of Physics: Condensed Matter, 27 (2015) 316301.

[47] A. Raj, J. Eapen, Radiation Damage Simulation in Graphite, Carbon, (Unpublished results).

[48] A. Chartier, L. Van Brutzel, B. Pannier, P. Baranek, Atomic scale mechanisms for the amorphisation of irradiated graphite, Carbon, 91 (2015) 395-407.

[49] H.J. Christie, M. Robinson, D.L. Roach, D.K. Ross, I. Suarez-Martinez, N.A. Marks, Simulating radiation damage cascades in graphite, Carbon, 81 (2015) 105-114.

[50] O.V. Yazyev, I. Tavernelli, U. Rothlisberger, L. Helm, Early stages of radiation damage in graphite and carbon nanostructures: A first-principles molecular dynamics study, Physical Review B, 75 (2007) 115418. 